\section{Diese Leistungen sind bei Asylsuchenden abrechenbar}

_ Im Asylbewerberleistungsgesetz ist für Asylsuchende, die sich noch keine 15 Monate in Deutschland aufhalten, nur ein eingeschränkter Anspruch auf medizinische Versorgung vorgesehen. Laut $\$ 4$ des Gesetzes gehören dazu die Behandlung akuter Erkrankungen und Schmerzzustände einschließlich der

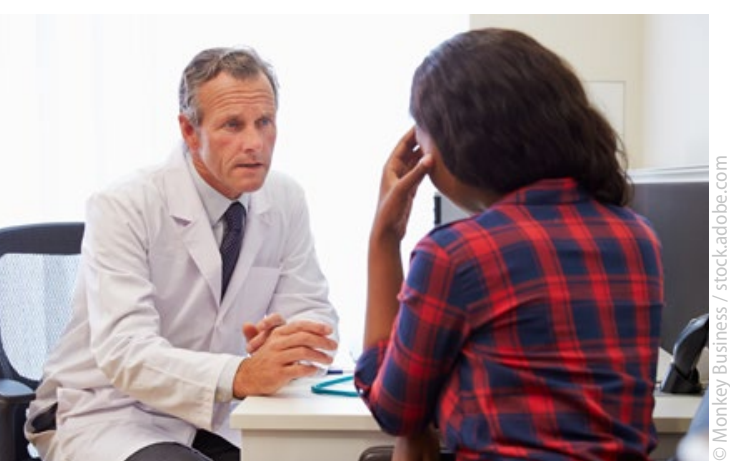

Akute Krankheiten können Asylsuchende jederzeit behandeln lassen.
Versorgung mit Arznei- und Verbandmitteln sowie sonstige Leistungen, die zur Genesung, Besserung oder Linderung von Krankheiten oder Krankheitsfolgen erforderlich sind.

Hinzu kommen Schutzimpfungen und Vorsorgeuntersuchungen zur Verhütung und Früherkennung von Krankheiten, wie sie im Abschnitt II.1.7.2 des EBM zusammengefasst sind. Werdende Mütter und Wöchnerinnen haben darüber hinaus Anspruch auf ärztliche und pflegerische Hilfe und Betreuung, Hebammenhilfe, Arznei-, Verband- und Heilmittel.

Weitere ärztliche Leistungen, z.B. eine fachärztlich kardiologische oder psychotherapeutische Behandlung, sind auch möglich, wenn sie zur Sicherung des Lebensunterhalts oder der Gesundheit unerlässlich sind. Eine entsprechende Überweisung an einen Spezialis-

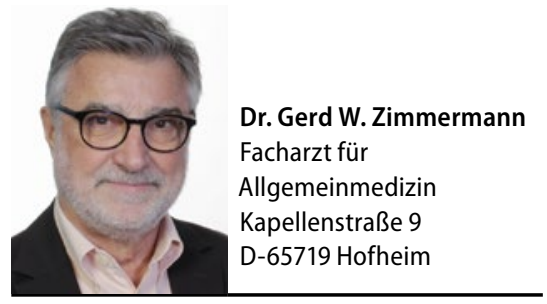

ten muss allerding vorher von der zuständigen Behörde genehmigt werden.

\section{MMW-KOMMENTAR}

Um in diesem Ausmaß medizinisch versorgt zu werden, müssen Asylsuchende als Behandlungsausweis einen von der örtlichen Behörde ausgestellten Behandlungsschein vorlegen. Seit Anfang 2016 können Länder und Kommunen zudem mit den gesetzlichen Kassen vereinbaren, eine elektronische Gesundheitskarte (eGK) an Asylsuchende auszugeben. Diese Karte hat im Feld "Besondere Personengruppe" die Ziffer 9 gespeichert. Daran kann die in Anspruch genommene Praxis erkennen, dass ein eingeschränkter Leistungsanspruch zu beachten ist. Ein optisches Zeichen oder einen sonstigen "Warnhinweis" auf der Karte selbst gibt es nicht. Um Regresse zu vermeiden, sollte diese Kennzeichnung deshalb unbedingt beachtet werden.

\title{
Testverfahren zusätzlich zu Geriatrieziffern abrechnen
}

_ Die Nr. 03242 EBM steht für Testverfahren bei Demenzverdacht, z. B. den Syndrom-Kurztest (SKT), den MiniMental-Status-Test (MMST) oder den Test zur Früherkennung von Demenzen mit Depressionsabgrenzung (TFDD). Die Nr. kann je Test bis zu dreimal im Behandlungsfall mit je 2 Euro abgerechnet werden. Eine Zeitvorgabe für die Plausibilitätsprüfung gibt es hier nicht.

\section{MMW-KOMMENTAR}

Beachtenswert ist, dass die Nr.03242 nur neben dem hausärztlich-geriatrischen Basis- assessment nach Nr.03360 im gesamten Behandlungsfall ausgeschlossen ist. Das bedeutet, dass eine parallele Berechnung neben dem hausärztlich-geriatrischen Betreuungskomplex nach Nr.03362 durchaus möglich ist.

Man sollte sich dabei vor Augen führen, dass die Leistung nach Nr. 03360 im Krankheitsfall - und damit innerhalb von vier Quartalen - höchstens zweimal berechnungsfähig ist. Das bedeutet, dass man das Basisassessment und die Testverfahren nach Möglichkeit ", auf Lücke" erbringen muss, wenn man jede Leistung abrechnen will.

\section{Neue Formulare im Januar}

Ab dem 1. Januar 2018 wird die Arbeitsunfähigkeitsbescheinigung (Muster 1) um Hinweise ergänzt. Auf dem Durchschlag für den Arbeitgeber wird vermerkt, dass die Krankenkasse „unverzüglich“ über die Arbeitsunfähigkeit informiert werden muss. Die Versicherten werden auf ihrem Durchschlag darauf hingewiesen, dass sie diesen „innerhalb von einer Woche" an die Kasse weiterleiten müssen. In der Vordruckvereinbarung wurde zudem die grundsätzliche Möglichkeit festgehalten, die Ausfertigung für die Krankenkasse elektronisch zu übermitteln.

Eine andere Änderung betrifft das Muster 18, die Heilmittelverordnung für Maßnahmen der Ergotherapie. Dieses Formular wird künftig auch für die Ernährungstherapie genutzt, die ab Januar verordnet werden kann. 